\title{
Forecasting the Number of Road Traffic Fatalities in Greece
}

\author{
Constantinos Antoniou ${ }^{1}$, George Yannis $^{2}$, Eleonora Papadimitriou ${ }^{3 *}$, Sylvain \\ Lassarre $^{4}$, Jacques F. Commandeur ${ }^{5}$, Frits Bijleveld ${ }^{6}$ and Emmanuelle Dupont ${ }^{7}$ \\ ${ }^{1}$ National Technical University of Munich, Germany \\ ${ }^{2,3}$ National Technical University of Athens, Greece \\ ${ }^{4}$ IFSTTAR - France \\ ${ }^{5,6}$ SWOV Institute for Safety Research, Netherlands \\ ${ }^{7}$ Belgian Road Safety Institute, Belgium \\ ${ }^{1}$ c.antoniou@tum.de, ${ }^{2}$ geyannis@central.ntua.gr, ${ }^{3}$ nopapadi@central.ntua.gr, \\ ${ }^{4}$ sylvain.lassarre@ifsttar.fr, ${ }^{5}$ jacques.commandeur@swov.nl, \\ 6frits.bijleveld@swov.nl, 7 emmanuelle.dupont@ibsr.be
}

\begin{abstract}
The objective of this research is the modelling and forecasting of road traffic fatalities in Greece for the period 2011-2020 by means of structural time series models. A dedicated methodology is used, developed within the DaCoTA research project of the European Commission, including Seemingly Unrelated Time Series models (SUTSE), Local Linear Trend models (LLT) and Latent Risk Models (LRT), as well as a set of decision criteria for selecting the most appropriate model. Fatality data for the period 1960-2010 are used, together with vehicle fleet data for the same period-due to the lack of the necessary vehicle-kilometres data. The SUTSE models developed as a first step suggested that the fatality and exposure series are unrelated, and therefore LLT models should be used on the fatality series alone. The best performing LLT model was found to be one with fixed slope and intervention variables to capture special events that took place at specific time points. The model was validated by using the last few observation as values to be predicted. Forecasts for the period 2011-2020 were then calculated, together with their confidence intervals. Actual data for years 2011 and 2012 fall within the confidence intervals range, however it should be kept in mind that these forecasts are affected by the economic recession visible at the end of the time series used, and which is expected to end sooner than 2020.
\end{abstract}

Keywords: structural time series models, forecasting, fatalities, exposure

\section{Background and Objectives}

During the last decade, the modeling approach of structural time-series models, such as those proposed by Harvey and Shephard [1] and Harvey [2], is applied by several researchers. In this approach, which belongs to the family of unobserved component models, latent variables are decomposed into components (hence the term "unobserved components"), which are incorporated into the structural models. Oppe $[3,4]$ modeled the latent traffic exposure and the latent risk of traffic fatalities in two separate steps. Lassarre [5] presented an analysis of ten European countries' progress in road safety by means of a structural (local line ar trend) model, yielding two adjusted trends, one deterministic and one stochastic. Stipdonk [6] applied multivariate analysis of the "three levels of risk" (i.e., exposure, fatality risk and accident severity) with structural time series models to quarterly data for the years 1987-2000 in France and the Netherlands, both at the national level, and stratified

${ }^{*}$ Corresponding Author 
by road type for France. Several researchers have focused on the LRT (Latent Risk model), in order to simultaneously model exposure and fatalities $[7,8,9]$.

More recently, the DaCoTA research project of the European Commission aimed to obtain forecasts for the number of traffic fatalities in each of the European countries in 2020 in a similar way by means of the structural time series approach, using comparable data as much as possible [10, 11,12]. A first objective was to develop robust models for modeling the relationship between mobility and risk and examine the effect of mobility on risk. A further objective was to develop (and apply) a structured methodology for the selection of the optimal forecasting models, based on a number of criteria, diagnostics and measures of goodness of fit.

The objective of this paper is to apply the DaCoTA methodology for the development of structural time series models for Greece, in order to forecast road traffic fatalities for the period 2011-2020. The paper starts with a presentation of the DaCoTA methodology for time series analysis and forecasting. Then, the time series data for Greece are presented and discussed. Subsequently, the models development process is described, towards the selection of the best performing model. Finally, the models are validated and the forecasts are discussed.

\section{Methodology}

Harvey and Sheppard [9] propose to decompose a univariate time-series $\mathrm{y}_{\mathrm{t}}$ into the following components:

$$
y_{t}=\mu_{t}+\psi_{t}+\gamma_{t}+\varepsilon_{t}
$$

where $\mu_{\mathrm{t}}$ is a trend, $\psi_{\mathrm{t}}$ is a cycle, $\gamma_{\mathrm{t}}$ is a seasonal and $\varepsilon_{\mathrm{t}}$ is an irregular component. All components are assumed stochastic (except for the mean, a zero mean is expected for the other components) with uncorrelated disturbances.

Two structural time series models are considered in this paper: (i) the local linear trend model and (ii) the latent risk time-series model [13]. Furthermore, a structured decision tree for the selection of the applicable model for each situation (developed within the DaCoTA research project) is outlined.

\subsection{Structural Time-Series Models: Local Linear Trend (LLT) and Latent Risk Time-Series (LRT) Models}

A basic concept in road safety is that the number of fatalities is a function of the road risk and the level of exposure of road users to this risk [4]. This implies that in order to model the evolution of fatalities it is required to model the evolution of two parameters: a road safety indicator and an exposure indicator:

$$
\begin{aligned}
& \text { Trafficvolume }=\text { Exposure } \\
& \text { Numberof fatalilties }=\text { Exposure } \times \text { Risk }
\end{aligned}
$$

which represents a latent risk time-series (LRT) formulation. In this case, both traffic volume and number of fatalities are treated as dependent variables. Effectively, this implies that traffic volume and fatality numbers are considered to be the realized counterparts of the latent variables "exposure", and "exposure x risk". When the logarithm of Equations 2 is taken (and the error term is explicitly written out) the -so called- measurement equations of the model can be rewritten as:

Log Traffic volume $=\log$ exposure + randomerrorintrafficvolume

Log Number of fatalities $=\log$ exposure $+\log$ risk + random errorof fatalities 
The latent variables [log (exposure) and $\log$ (risk)] need to be further specified by "state" equations, which, once inserted in the general model, describe the development of the latent variable. Equations (4) and (5) show how a variable can be modeled (to simplify the illustration only the number of fatalities is decomposed as an example):

Measurement equation:

$$
\log \text { Number of Fatalities }_{t}=\log \text { LatentFat }_{t}+\varepsilon_{t}
$$

State equations:

$$
\begin{aligned}
& \text { Level }\left(\log \text { LatentFat }_{t}\right)=\operatorname{Level}\left(\log \text { LatentFat }_{t-1}\right)+\text { Slope }\left(\log \text { LatentFat }_{t-1}\right)+\xi_{t} \\
& \text { Slope }\left(\log \left(\text { LatentFat }_{t}\right)=\text { Slope }\left(\log \text { LatentFat }_{t-1}\right)+\zeta_{t}\right.
\end{aligned}
$$

A more general formulation is presented in Equation (6), in which $Y_{t}$ represents the observations and is defined by the measurement equation within which $\mu_{t}$

represents the state and $\varepsilon_{t}$ the measurement error. The state $\mu_{t}$ is defined in the state equation, which essentially describes how the latent variable evolves from one time point to the other.

$$
\begin{aligned}
& Y_{t}=\mu_{t}+\varepsilon_{t} \\
& \mu_{t}=\mu_{t-1}+v_{t-1}+\xi_{t} \\
& v_{t}=v_{t-1}+\zeta_{t}
\end{aligned}
$$

In the present case, the state $\mu_{t}$ thus corresponds to the fatality trend at year $t$. It is defined by an intercept, or level $\mu_{t-1}$ (thus the value of the trend for the year before, assuming an annual time-series) plus a slope $v_{t-1}$, which is the value by which every new time point is incremented (or decremented depending on the slope sign, which is usually negative in the case of fatality trends). The slope $v_{t}$ thus represents the effect of time on the latent variable. It is defined in a separate equation, so that a random error term can be added to it $\left(\zeta_{t}\right)$. These random terms, or disturbances, allow the level and slope coefficients of the trend to vary over time.

The basic formulation presented in Equation (6) allows the definition of a rich family of trend models which covers an extensive range of series in a coherent way; when both the level and slope terms are allowed to vary over time the resulting model is referred to as the local linear trend (LLT) model.

The next model is a Latent Risk Time-Series (LRT), which simultaneously models exposure and fatalities. To accomplish this, the latent risk model contains two measurement equations: one for the exposure (e.g., traffic volume) and one for the fatalities; two state equations can be written for each measurement equation, modeling the level and slope of the corresponding latent variable.

For traffic volume:

Measurement equations:

$\log$ Traffic Volume $_{t}=\log$ Exposure $_{t}+\varepsilon_{t}{ }^{e}$

State equations: 


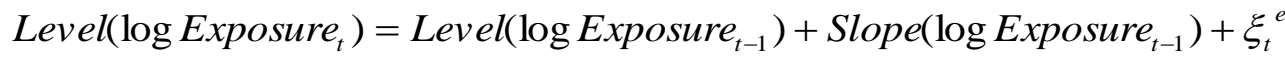

Slope $\left(\log\right.$ Exposure $\left._{t}\right)=$ Slope $\left(\log\right.$ Exposure $\left._{t-1}\right)+\zeta_{t}{ }^{e}$

For the fatalities:

Measurement equation:

$\log$ Number of Fatalities $_{t}=\log$ Exposure $_{t}+\log$ Risk $_{t}+\varepsilon_{t}{ }^{f}$

State equations:

$\operatorname{Trend}\left(\log \operatorname{Risk}_{t}\right)=\operatorname{Level}\left(\log \operatorname{Risk}_{t-1}\right)+\operatorname{Slope}\left(\log \operatorname{Risk}_{t-1}\right)+\xi_{t}{ }^{r}$

Slope $\left(\log \operatorname{Risk}_{t}\right)=$ Slope $\left(\log \operatorname{Risk}_{t-1}\right)+\zeta_{t}{ }^{r}$

Note that Equation (9) now includes the Risk (and not the fatalities), which can be estimated as:

$\log$ Risk $_{\mathrm{t}}=\log$ LatentFat $_{\mathrm{t}}-\log$ Exposure $_{\mathrm{t}}$

Seemingly Unrelated Time-Series Equations (SUTSE) [1], a third class of models, are also used in this approach as a preliminary step in establishing whether the two time-series may be correlated.

\subsection{Model Selection Criteria}

The family of structural time-series models lends to a large number of assumptions that distinguish the resulting models into different categories. Within the framework of the DACOTA research project, a decision process and model selection logic has been developed, in which the following steps are considered:

- Investigate exposure: the first step in every modeling effort is to assess the quality and characteristics of the underlying data. Do the available exposure data make sense? Can any sudden changes in the level or slope be explained from some real events?

- Establish whether the two series are statistically related: a SUTSE model is developed and based on the diagnostics, the modeler needs to decide whether the two time-series are correlated.

- Depending on the output of the SUTSE model determine whether an LLT or an LRT model should be pursued: If one or more of the null-hypotheses regarding the correlation of the disturbances is rejected, the time-series may be related and therefore an LRT can be estimated. If, on the other hand, none of the hypotheses can be rejected, then there is no evidence that the two time-series are correlated and therefore an LLT model would be more appropriate.

\section{Data Description}

\subsection{Exposure}

It is widely accepted that vehicle kilometres are an appropriate exposure measure. However, there are no vehicle kilometres data available for Greece and therefore the vehicle fleet is used as a proxy. The selected exposure measure are the vehicles in circulation (in thousands) per annum (see Figure 1), which are collected by the Ministry of Infrastructure, Transport and Networks, and are here considered from 1960 onwards. 


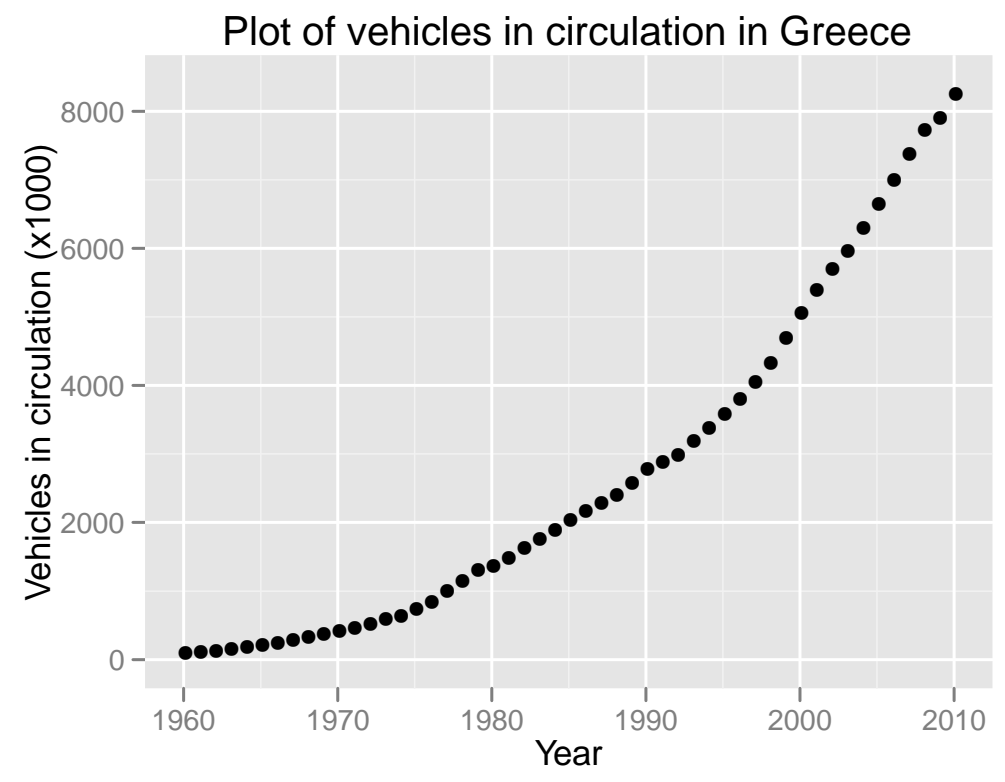

Figure 1. Plot of the Annual Vehicle Fleet (x1000) for Greece from 1960 to 2010

The number of vehicles in circulation continuously increased from 1960 to almost 2008. During the last couple of years, the rate of increase appears to slow down, reflecting the effect of the recession. However, this effect is not as evident as it would be if a more appropriate measure of exposure, such as vehicle-kilometres, was available. If it was available, the series of vehicle-kilometres would actually show a reduction in the years of recession, and not simply a reduced increase. Compared to vehicle-kilometres, the number of vehicles is a less volatile measure of exposure, as (i) a reduction in the use of the vehicles does not necessarily correspond to a reduction of the number of vehicles and (ii) even when the vehicles are removed from the circulation; it is not as easy to update the registry of vehicles.

\subsection{Fatalities}

The Greek road accident fatality figures from 1960 to 2010 are plotted in Figure 2. The data are collected by the Traffic Police, and gathered, processed and stored by the Hellenic Statistical Authority (ELSTAT). Before 1996 road accident fatalities in Greece were recorded based on the 24-hour definition (i.e., only the persons who passed away within 24 hours from the occurrence of the accident were defined as road safety fatalities and registered as such). Since 1996 the 30-day definition is used. The data presented in Figure 2 correspond to the 30-day definition for the entire period (converted via appropriate factors for the period prior to 1996).

The fatality data for Greece shows two distinct trends: an increasing one until approximately 1995 , followed by a decreasing one thereafter. 


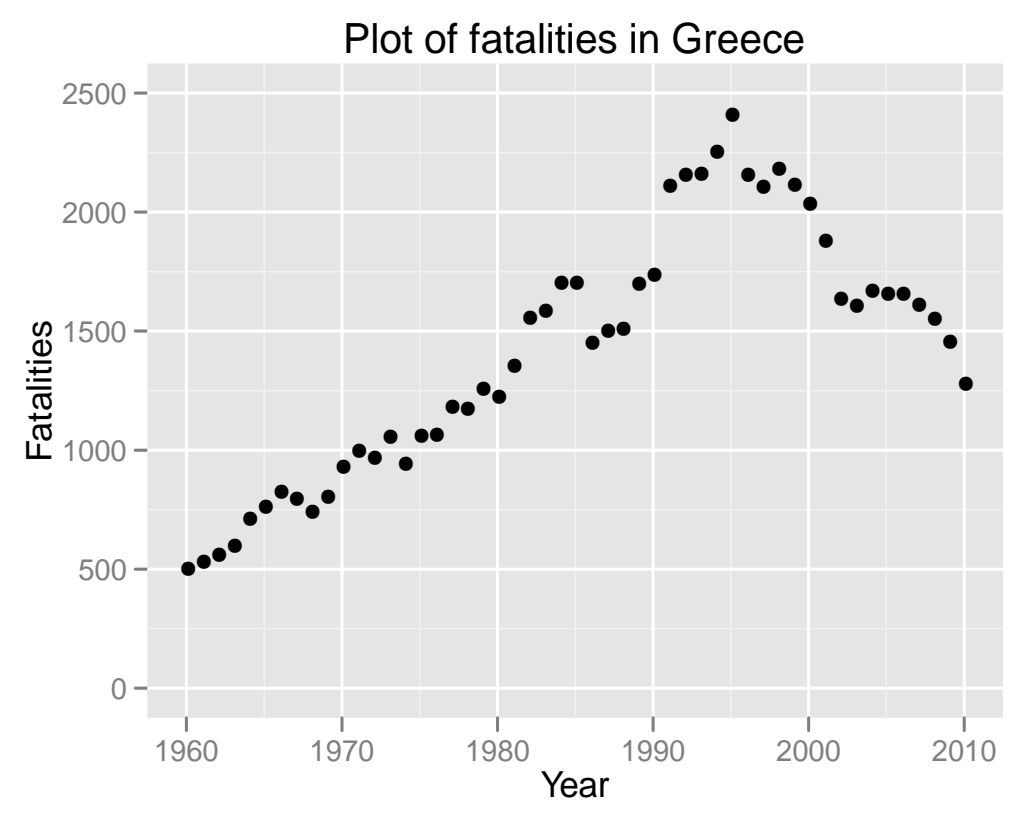

Figure 2. Plot of the Annual Fatality Counts for Greece from 1960 to 2010

While the exposure data seem rather smooth, the fatality data exhibit certain irregularities that could affect the model estimation results. In order to better account for these external shocks to the process, it was decided to seek possible events that could be identified and explicitly entered into the model. There are three main events that can be entered as interventions in the model for the period and data that are being analysed:

- I1986: in 1986 Greece encountered a financial crisis, which affected mobility and therefore exposure. This intervention is entered into the model as a shock in the specific time point at the level of the exposure.

- I1991: in 1991, Greece introduced a "car scrappage" scheme, under which old cars could be exchanged for a cash incentive to buy a new (safer and cleaner) car. This intervention is also entered into the model as a shock in the specific time point at the level of the exposure.

- I1996: in 1996 the fatality recording system in Greece switched from 24-hour to 30-day. This means that the adjustment factor (from 24-hour to 30-day fatality figures) stopped to be used at that time and that real data was used from that point on. This intervention has been entered in the slope of the fatalities, as its impact is assumed to be unlike a point shock, but rather a sustained shift.

Additional interventions were also considered within the examined period, but were not found to be significant (e.g., the $1^{\text {st }}$ and the $2^{\text {nd }}$ Strategic Plan for Road Safety in Greece, which were adopted but not fully implemented).

\section{Models Development}

\subsection{The SUTSE Model}

Figure 3 presents the varying level and slope estimation results of the SUTSE model: in particular the smoothed state plots for the exposure (top) and risk (bottom) variables. Confidence intervals are also presented in these figures. The confidence intervals on the 
levels are rather tight and are closely following the trends. What is perhaps more interesting is the slope of the variables. The slope of the exposure (top right subfigure) is always positive, but its magnitude is declining. The slope of the risk (bottom right subfigure) is also decreasing.

\section{Exposure}

While the trend component is fairly smooth, and does not vary significantly over time. The number of vehicles in circulation in Greece increased from less than $100 \mathrm{~K}$ in 1960 to more than 8 million in 2010. The slope values vary significantly, indicating that this increase did not take place at a constant rate. In particular, the rate of increase in the early 1960 s was about $17 \%$, while it has fallen below $5 \%$ in the recent years.

\section{Fatalities}

The level component varies significantly, and so does the slope although to a lesser extent. The most important feature of the level component is a break in the trend from increasing to decreasing in 1995. The fatalities increased from about 500 in 1960 to about 2300 in 1995, and then dropped to about 1300 in 2010. In terms of the slope, the increase pretty much constantly reduced from about 5\% in 1960 to zero in 1995 and then continuously decreased until about $3.5 \%$ in 2010.

\section{Correlation between the disturbances of the state components}

Two state components, the level of exposure cannot be considered stochastic, while the slope of the fatalities, is significant in the SUTSE model but insignificant in the SUTSE/beta model, implying that the slope of the fatalities may be stochastic. The correlation between the two levels $(\mathrm{p}=0.33)$ and two slopes $(\mathrm{p}=0.77)$ is not significant. The value of the correlation is 0.35 between the two levels and 0.24 between the two slopes.

\section{Correlation between the irregulars}

The measurement errors for exposure and fatalities are correlated at $6.4 \mathrm{E}-05$ which is not significant $(\mathrm{p}=1)$.

\section{Estimation of the relationship by means of a coefficient}

The relation between exposure and fatalities estimated by the beta coefficient in a restricted SUTSE/LRT model is 0.45 and is not significant ( $\mathrm{p}=0.34)$.

Furthermore, the log-likelihood for the two models is very similar, indicating that a possible time-varying relation between exposure and fatalities is unlikely. Therefore, it can be concluded that the fatalities and vehicle fleet series are not related. In such a case, it is not advisable to use the exposure indicator (in this case, vehicle fleet) to estimate the fatality risk and run an LRT model. As a consequence, an LLT model was used. 


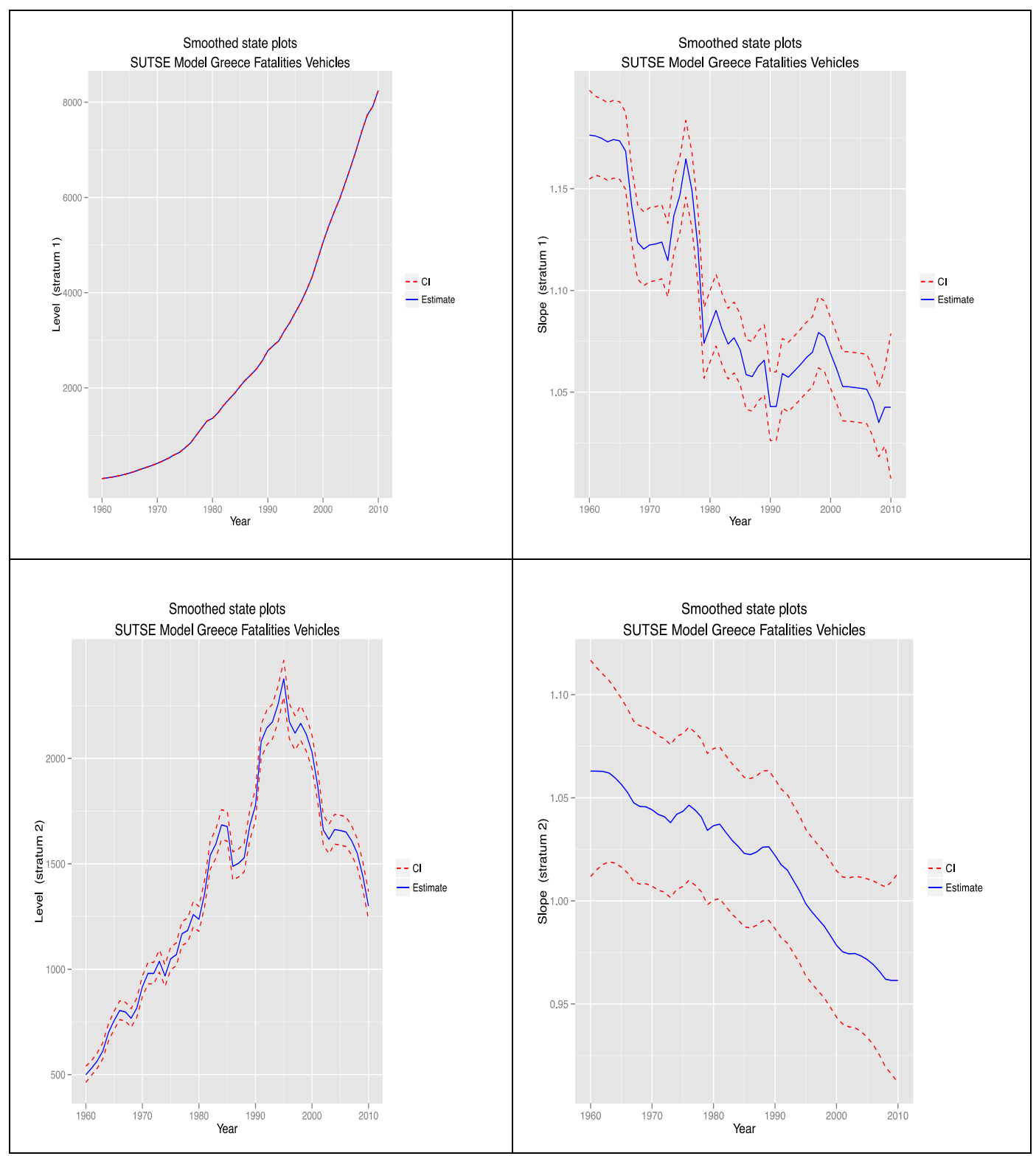

Figure 3: Greece - Developments of the State Components for the Exposure (Upper Graphs) and the Fatalities (Lower Graphs), as Estimated on the Basis of the SUTSE Model. The Trend (Level) Developments are Represented in the Right-hand Graphs, the Slope Developments in the lefthand Graphs 
Table 1. Overview of the Results for SUTSE Models - Greece

\begin{tabular}{|c|c|c|}
\hline Model title & SUTSEGreece1 & SUTSEbetaGreece1 \\
\hline Model description & SUTSE full model & $\begin{array}{l}\text { SUTSE independent } \\
\text { components, beta } \\
\text { estimated }\end{array}$ \\
\hline \multicolumn{3}{|l|}{ Model Criteria } \\
\hline log likelihood & 237.76 & 237.42 \\
\hline AIC & -475.17 & -474.53 \\
\hline \multicolumn{3}{|l|}{ Variance of the state components } \\
\hline Level exposure & $1.33 \mathrm{E}-04 \mathrm{nsc}$ & $1.23 \mathrm{E}-04 \mathrm{~ns}$ \\
\hline Level fatalities & $4.06 \mathrm{E}-03 * \mathrm{c}$ & $3.88 \mathrm{E}-03 *$ \\
\hline Slope exposure & $2.17 \mathrm{E}-04 * \mathrm{c}$ & $2.09 \mathrm{E}-04 *$ \\
\hline Slope fatalities & $1.09 \mathrm{E}-04 * \mathrm{c}$ & 7.43E-05 ns \\
\hline $\begin{array}{l}\text { Correlations between the state } \\
\text { components }\end{array}$ & & \\
\hline level-level & 0.35 & 1 \\
\hline slope-slope & 0.24 & 1 \\
\hline \multicolumn{3}{|l|}{ Observation variance } \\
\hline Observation variance exposure & $1.014 \mathrm{E}-09 \mathrm{~ns}$ & $5.16 \mathrm{E}-06 \mathrm{~ns}$ \\
\hline Observation variance fatalities & $1.689 \mathrm{E}-09 \mathrm{~ns}$ & $9.01 \mathrm{E}-05 \mathrm{~ns}$ \\
\hline Beta & & $0.45 \mathrm{~ns}$ \\
\hline
\end{tabular}

\subsection{The LLT Model}

The investigation of the SUTSE model indicates that a relation between vehicle fleet and fatalities in Greece is not present. Therefore, an LLT model is fit for Greece.

Three versions of the LLT model were run. The full model (LLT1) was run first. None of the residual tests indicated a violation of the underlying assumptions. Furthermore, the level and slope components were significant. Therefore, a new model (LLT2) with additional interventions was estimated. While the fit of this model improved compared to the original model, the slope component became insignificant. Therefore, a third model (LLT3) was also run, with the interventions, but keeping the slope of the fatalities fixed. 
The incorporation of the three interventions in the model LLT2 led to a considerable improvement over the model LLT1 both in terms of log-likelihood and AIC, but also in terms of quality of the predictions. As discussed above, considering the small number of data points following the change in fatality trends, only results with 4 observations held out for validation are presented. The ME and MSEs values based on these last 4 years are lower when based on LLT2 and 3 than when based on the full model. This suggests that the model is better able to predict the observations once the interventions are introduced. Model LLT3 has one more degree of freedom over LLT2 (since the slope of the fatalities is fixed) and both the log-likelihood and AIC, as well as the residual statistics ME and MSE improve. Therefore, this model is selected.

Table 2. Overview of the Results for LLT Models - Greece

\begin{tabular}{llll}
\hline Model title & LLT 1 & LLT 2 & \multicolumn{2}{l}{ LLT3 } \\
& & LLT for \\
Model description & Greece - \\
& LLT for Greece - Greece - for with interventions \\
& full model & 3 interventions & - fixed slope
\end{tabular}

Model Criteria

\begin{tabular}{llll}
\hline ME4 Fatalities & -131 & -61.4 & -59.4 \\
MSE4 Fatalities & 28162.3 & 10047.9 & 9689.6 \\
\hline log likelihood & 85.66 & 65.84 & 65.82 \\
AIC & -171.21 & -131.56 & -131.55 \\
\hline
\end{tabular}

Model Quality

\begin{tabular}{llll}
\hline Box-Ljung test 1 Fatalities & 2.73 & 2.96 & 0.29 \\
Box-Ljung test 2 Fatalities & 3.63 & 4.30 & 2.78 \\
Box-Ljung test 3 Fatalities & 5.82 & 4.33 & 4.03 \\
\hline Heteroscedasticity Test Fatalities & 0.79 & 0.75 & 0.76 \\
\hline $\begin{array}{l}\text { Normality Test standard Residuals } \\
\text { Fatalities }\end{array}$ & 0.80 & 1.95 & 2.06 \\
\hline Normality Test output Aux Res Fatalities & 1.28 & 1.13 & 1.17 \\
\hline $\begin{array}{l}\text { Normality Test State Aux Res Level risk } \\
\text { Normality Test State Aux Res Slope risk }\end{array}$ & 1.61 & 1.34 & 1.10 \\
& 0.05 & 0.00 & 0.00 \\
Variance of state components & & & \\
\hline $\begin{array}{l}\text { Level risk } \\
\text { Slope risk }\end{array}$ & $3.91 \mathrm{E}-03 *$ & $2.61 \mathrm{E}-03 *$ & $2.67 \mathrm{E}-03 *$ \\
& $1.25 \mathrm{E}-04 *$ & $6.92 \mathrm{E}-06 \mathrm{~ns}$ & -
\end{tabular}




\begin{tabular}{|c|c|c|c|}
\hline Observation variance risk & $1.00 \mathrm{E}-09 \mathrm{~ns}$ & $1.00 \mathrm{E}-09 \mathrm{~ns}$ & $1.00 \mathrm{E}-09 \mathrm{~ns}$ \\
\hline \multicolumn{4}{|l|}{$\begin{array}{l}\text { Intervention and explanatory variables } \\
\text { tests }\end{array}$} \\
\hline \multicolumn{4}{|l|}{ Change in fatality recording system } \\
\hline (slope fat 1996) & & $-0.074 *$ & $-0.080 *$ \\
\hline Financial crisis (level fat 1986) & & $-0.209 *$ & $-0.211 *$ \\
\hline $\begin{array}{l}\text { Introduction of car scrappage system (level } \\
\text { fat 1991) }\end{array}$ & & $0.152 *$ & $0.147 *$ \\
\hline
\end{tabular}

\subsection{Quality of the Predictions}

As there are only 15 data points describing the decreasing trend, it is expected that reserving a large number of observations for forecasting may affect the accuracy of the model. To evaluate how well models implemented here have done in the past, the data up to 2006 are used to forecast the fatalities between 2007 and 2010. This rather short number of observations is selected based on the nature of the last few observations and the overall nature of the fatality data (with the breakpoint in 1995). A larger number of observations reserved for validation, would leave a smaller number of observations for the model to capture the breakpoint and downward trend in the recent years). Figure 4 below shows a comparison between the predicted and actually observed values for the estimated models. The results shown in Figure 4 indicate that the model with the interventions result in much better predictions than the model without interventions.

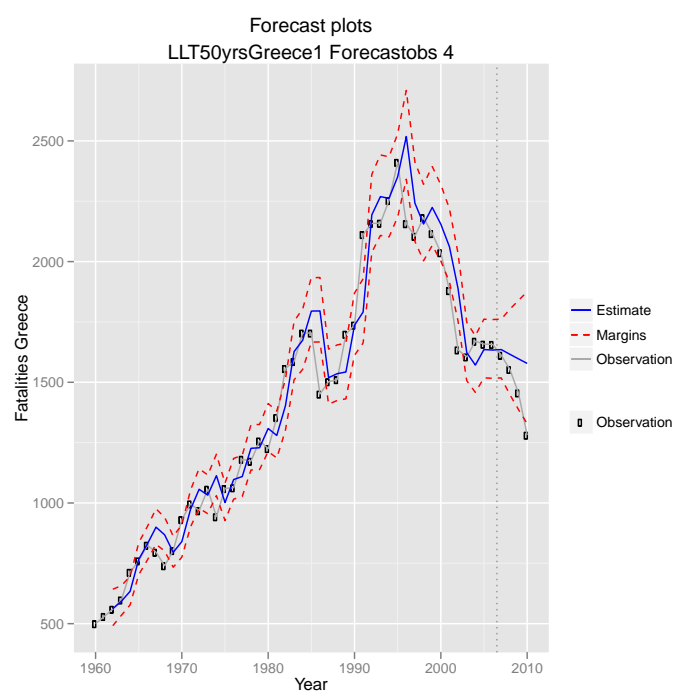




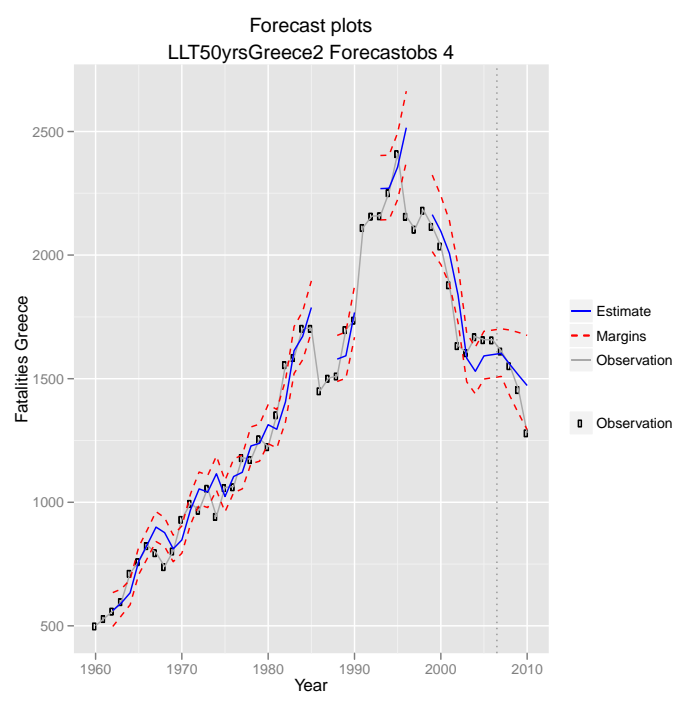

Figure 4. Plots Comparing the Model Predictions (Straight Line) with the Actual Observations ("bullets") for the Annual Fatality Numbers in Greece

\section{Forecasts}

The forecasts in Figure 5 and Table 3 provide an indication of the fatality numbers that could be expected in Greece between 2011 and 2020 provided that the current trends keep on following throughout these years.

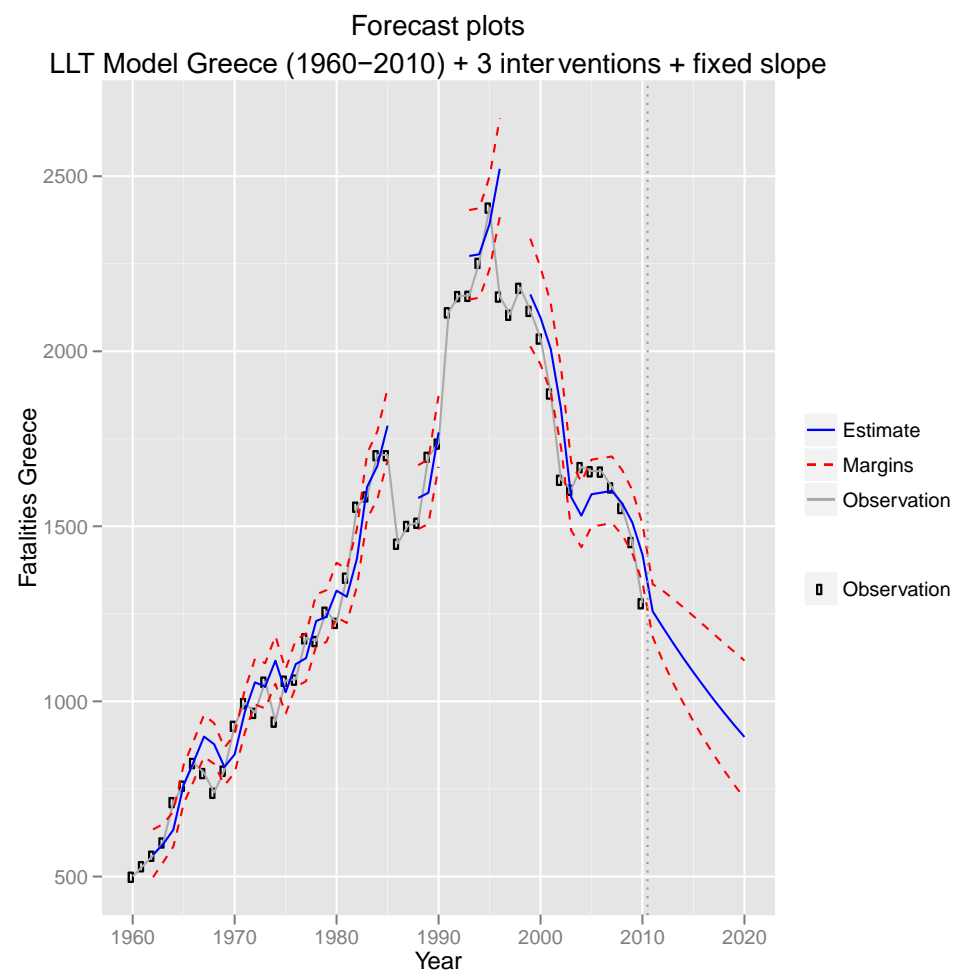

Figure 5. Forecast Values for 2011-2020 for Greece Based on the Selected Local Linear Trend Model with Interventions and Fixed Slope 
Table 3. Forecasts of Local Linear Trend (LLT3) for Greece

\begin{tabular}{cccc}
\hline \multicolumn{3}{c}{ Fatalities } \\
Year & $\begin{array}{c}\text { Predic } \\
\text { ted }\end{array}$ & $\begin{array}{c}\text { Confidence } \\
\text { Interval }\end{array}$ \\
2011 & 1257 & 1118 & 1414 \\
2012 & 1211 & 1029 & 1426 \\
2013 & 1167 & 953 & 1429 \\
2014 & 1124 & 885 & 1427 \\
2015 & 1083 & 824 & 1422 \\
2016 & 1043 & 769 & 1415 \\
2017 & 1005 & 717 & 1407 \\
2018 & 968 & 670 & 1398 \\
2019 & 932 & 626 & 1389 \\
2020 & 898 & 585 & 1379 \\
\hline
\end{tabular}

\section{Discussion}

The present research applied a methodological framework for forecasting road safety developments with structural time series models [11]. The proposed methodology was proved, within DaCoTA, to be very efficient for handling different cases of data availability and quality, providing an appropriate alternative from the family of structural time series models in each case. The estimated forecasts in all European countries appear to be realistic and within acceptable confidence intervals. The forecasts are based on "business-as-usual" scenarios. The decision to include intervention variables was based on the availability of information on specific interventions or events (road safety related or socio-economic).

Different exposure measures were available in different countries, ranging from the most appropriate ones, i.e. passenger and vehicle-kilometres, to the "second best", i.e., fuel consumption, to the less appropriate, i.e., vehicle fleet. The example of Greece seems to confirm the limited usefulness of vehicle fleet data as a proxy of exposure, as it was proved to be not at all related with road safety developments.

A range of models from the family of structural time series models were tested, and a local linear trend (LLT) model with interventions was proved to be the best model for describing and forecasting fatalities in Greece. It is noteworthy that the implementation of a simple LLT model results in a 35\% confidence level; the fact that without interventions the results are quite inaccurate may suggest that the prognosis period should not be too long. From the best fitting model, road safety forecasts were made, and their 95\% confidence intervals were calculated. The confidence intervals, and in particular their width, are a reflection of the uncertainty/variability of the time-series.

In order to validate the forecasting performance of the models, we have used observed fatality data from the last few years (2010-2012). Table 4 presents these true fatality figures for Greece along with the corresponding model forecasts for these years (along with the $95 \%$ confidence intervals). The true fatality figures fall within (or right on) the $95 \%$ confidence intervals. 
Table 4. Overview of Short-term Fatality Forecasts and True Values

\begin{tabular}{l|clcc}
\hline \multicolumn{1}{c}{ Greece } & \multicolumn{3}{l}{} \\
\hline Year & $\begin{array}{c}\text { Forecast } \\
\text { fatalities }\end{array}$ & $\begin{array}{l}95 \% \text { conf. interval } \\
\text { (from }- \text { to) }\end{array}$ & $\begin{array}{c}\text { Actual } \\
\text { fatalities }\end{array}$ \\
2011 & 1257 & 1118 & 1414 & 1141 \\
2012 & 1211 & 1029 & 1426 & 1027 \\
\hline
\end{tabular}

It may be particularly important to note that in Greece the economic recession effect is visible at the end of the fatalities series, which in turn affects the final forecasts. A scenario in which the forecasted value for 2020 is somewhat increased, may in this case provide a more realistic picture of future developments, as it takes into account the fact that the recession will end sooner (while in the baseline "business-as-usual" scenario, the effect of the recession is assumed to continue in the future) [14].

\section{Acknowledgments}

This paper is based on work carried out within the scope of the DaCoTA (Data Collection Transfer and Analysis) project of the 7th Framework RTD Program of the European Commission. The authors would like to acknowledge the important contribution of all the partners involved in the DaCoTA WP4 "Decision Support" in the development of the methodologies and outputs used in this paper.

\section{References}

[1] A. C. Harvey and N. Shephard, "Structural Time Series Models", In Maddala GS, Rao CS, Vinod HD, eds. Handbook of Statistics. Elsevier Science Publishers, B.V., vol. 11, (1993), pp. 261-302.

[2] A. C. Harvey, "Forecasting, Structural Time Series Models and the Kalman Filter", Cambridge: University Press, (1994).

[3] S. Oppe, "Macroscopic Models for Traffic and Traffic Safety", Accid Anal Prev., vol. 21, (1989), pp. 225-232.

[4] S. Oppe, "Development of traffic and traffic safety: global trends and incidental fluctuations", Accident Analysis and Prevention, vol. 23, no. 5, (1991), pp. 413-22.

[5] S. Lassarre, "Analysis of progress in road safety in ten European countries", Accid Anal Prev., vol. 33, (2001), pp. 743-751.

[6] H. L. Stipdonk, (ed.), "Time series applications on road safety developments in Europe", Deliverable D7.10 of the EU FP6 project SafetyNet; (2008).

[7] F. Bijleveld, J. Commandeur, P. Gould and S. J. Koopman, "Model-based measurement of latent risk in time series with applications", Journal of Royal Stat Soc A., vol. 171, (2008), pp. 265-277.

[8] COST 329, "Models for traffic and safety development and interventions", European Commission, Directorate general for Transport, Brussels. http://www.cost.eu/media/publications/04-16-Models-forTraffic-and-Safety-Development-and-Interventions-Final-Report, (2004).

[9] F. Van den Bossche, G. Wets and T. Brijs, "Analysis of road risk per age and gender category: A time series approach", Transportation Research Record - Journal of the Transportation Research Board, 20197-14, (2007).

[10] Martensen \& Dupont (Eds.), "Forecasting road traffic fatalities in European countries: model and first results. Deliverable 4.2 of the EC FP7 project DaCoTA", http://www.dacotaproject.eu/Deliverables/DaCoTA_WP4_D4_2_Final\%20after\%20Revision.pdf, (2010).

[11] Dupont \& Martensen (Eds.), "Forecasting road traffic fatalities in European countries: model and first results. Deliverable 4.4 of the EC FP7 project DaCoTA", http://www.dacotaproject.eu/Deliverables/DaCoTA_D4_4\%20Final2.pdf, (2012).

[12] S. Lassarre (ed.), "Forecasting road traffic fatalities in European countries", Deliverable 4.7 of the EC FP7 project DaCoTA; (2012).

[13] F. Bijleveld, "Time series analysis in road safety research using state space methods", Amsterdam, Netherlands: Free University, Doctoral Dissertation, (2008).

[14] C. Antoniou and G. Yannis, "State-space based analysis and forecasting of macroscopic road safety trends in Greece", Accident Analysis and Prevention, vol. 60, (2013), pp. 268-276. 